\title{
El marco de las inteligências múltiples, uma perspectiva para la profesionalización del perfil mediador
}

\author{
The framework of multiple intelligences, a perspective for the \\ professionalization of the mediator profile
}

\author{
Ivan Sena-Urdiales ${ }^{1}$ \\ Karla Sáenz ${ }^{2}$ \\ Enrique Pastor-Seller ${ }^{3}$
}

\begin{abstract}
RESUMEN
El presente documento tiene como fin proponer el marco de las Inteligencias Múltiples, teoría desarrollada por Howard Gardner, para estructurar el perfil profesional del mediador, con especial atención en el ámbito de la mediación familiar por su relevante carga emocional. Lo anterior se fundamenta en la idea de que es imposible decir que un campo del conocimiento predomina en la mediación ya que el ejercicio profesional tiene un carácter multi e interdisciplinario. Considerando estudios que recopilan la opinión de usuarios y la aplicación de una entrevista cualitativa a profesionales, se realiza la propuesta dentro del marco de las IM.
\end{abstract}

\section{PALABRAS CLAVE:}

Mediador, Mediación, Inteligencias Múltiples.

\section{ABSTRACT}

The purpose of this document is to propose the framework of Multiple Intelligences, a theory developed by Howard Gardner, to structure the professional profile of the mediator, with special attention in the field of family mediation due to its relevant emotional charge. This is based on the idea that it is impossible to say that a field of knowledge predominates in mediation since professional practice has a multi and interdisciplinary nature. Considering studies that compile the opinion of users and the application of a qualitative interview to professionals, the proposal is made within the framework of the IM.

\section{KEYWORDS:}

Mediator, Mediation, Multiple Intelligences.

\footnotetext{
${ }^{1}$ Doctorando en el Doctorado en Métodos Alternos de Solución de Conflictos de la Universidad Autónoma de Nuevo León (UANL), México, Maestro en Psicología con orientación en Psicología Laboral y Organizacional (UANL), Licenciado en Psicología (UANL). ivansenau@gmail.com.

${ }^{2}$ Doctora en Ciencia Política, catedrática de la Facultad de Contaduría Pública y Administración de la Universidad Autónoma de Nuevo León. Investigadora del Sistema Nacional de Investigadores Nivel 1 del Consejo Nacional de Ciencia y Tecnología. karla.saenzlp@uanl.edu.mx.

${ }^{3}$ Doctor en Trabajo Social. Catedrático de la Universidad de Murcia. Investigador reconocido epastor@um.es.
} 


\section{INTRODUÇÃO}

En la actualidad la mediación se ha considerado como un ejercicio multi e interdisciplinario, normalizando la idea de que un campo de conocimiento en específico no puede responsabilizarse de la total preparación de los profesionales que ejercen como mediadores en los procedimientos.

Al igual que las normativas, los campos de conocimiento presentan sus avances conforme al seguimiento de los resultados de las investigaciones, los métodos alternos de solución de conflictos no son la excepción; así como existe una normativa para el profesional de mediación, el presente documento busca ampliar en ese campo en específico, el perfil del mediador.

Iglesias-Ortuño (2016) afirma que la formación en mediación ha tenido un desarrollo mayor en comparación con los parámetros o estándares para controlar su pertinencia, calidad y resultados, y que hace falta prestar atención a los resultados de la formación en mediación a través de la competencia profesional con respecto a demanda social actual.

Gorjón menciona que la mediación como profesión "adolece de estudios específicos, ya que hasta el momento histórico la mediación y los MASC no han sido considerados como una profesión y no se centran en el mediador como un profesional” (2015, pág. 18). Aunque se considera que cuando una mediación fracasa es responsabilidad del facilitador y que si llega al éxito es gracias a las partes, el facilitador es quien ha sido el guía durante todo el procedimiento y por esto se debe considerar su influencia en el éxito del mismo. Por lo que nos parece de vital importancia explorar el aspecto profesional del facilitador de mediación en materia familiar desde un enfoque que nos permite comprender la relevancia que tiene su actuar en el procedimiento de mediación.

Aportes realizados por Serrano en su artículo "Eficacia y Mediación Familiar" propone un Modelo Integrador de Mediación Eficaz (MIME) (2008, pág. 58), en el cual describe los elementos que inciden en el resultado del procedimiento de mediación y donde menciona que "resulta muy expresivo que la mayor parte de las características de los mediadores asociadas 
con el éxito de la mediación haga referencia a lo que conocemos como "habilidades sociales" “ (2008, pág. 56), de acuerdo a la opinión de los participantes de procedimientos de mediación en investigaciones previas. Por esta razón centramos el interés del presente estudio con la intención de considerar la perspectiva de los mismos profesionales que ejercen como mediadores agregando un marco que incluye las competencias como elemento estructural para su descripción, el marco de las Inteligencias Múltiples (IM) de Howard Gardner (Estructuras de la mente, 2001).

Es importante considerar cuál es la salida de un proceso para poder hacer una medición adecuada de la eficiencia con la que se utilizan los recursos, un registro de qué tan eficaz es el proceso para así, posteriormente, analizar la efectividad que tiene el mismo. Para eso hay que tener en claro como aplica cada uno de estos tres conceptos en la mediación. De acuerdo a un estudio realizado por la INDES entre los años 1997 y 1998, donde participaron 262 profesionales en el diseño y gerencia de programas y políticas sociales, en donde el $84 \%$ de las respuestas coincidían en que "la eficacia corresponde al cumplimiento de metas y objetivos o a la satisfacción de necesidades que motivó el diseño y desarrollo de la iniciativa, sin importar los costos y el uso de recursos" (2000, pág. 3). Relacionado con lo propuesto por Serrano quien dice que "la mediación estructuralmente considerada no persigue prima facie la mejora de las relaciones sino la resolución del conflicto" (2008, pág. 55) lo cual se ve plasmado en la generación del acuerdo.

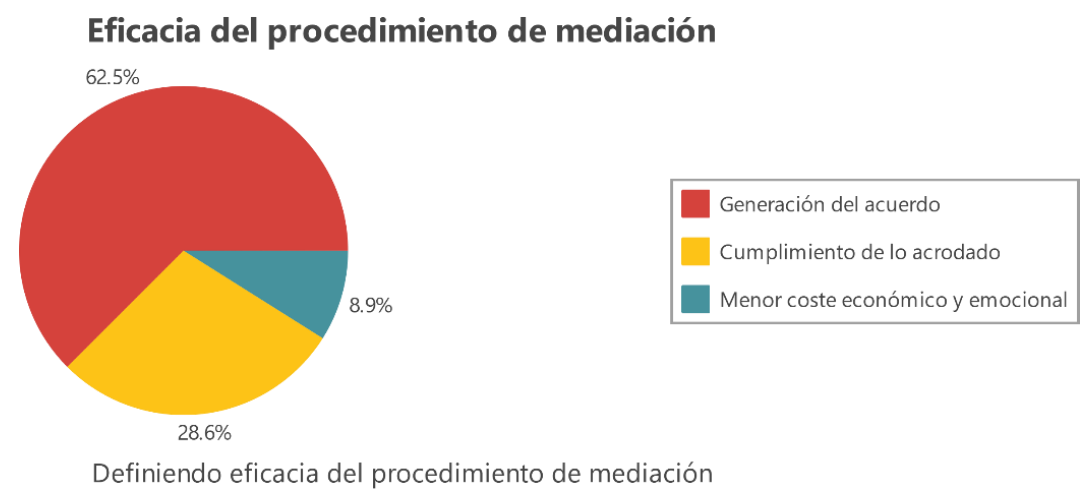

De acuerdo a la mayoría de las respuestas de los profesionales esta aproximación a la eficacia de la mediación es la apropiada puesto que consideran que hasta la generación del acuerdo es donde finaliza la incidencia del ejercicio profesional de la mediación y el procedimiento mismo ya que, aunque depende de las partes la generación del conflicto, el 
mediador es quien procura las condiciones dentro del procedimiento con la finalidad de lograr el acuerdo.

A través del presente documento se dará una introducción breve a la definición de Mediación haciendo especial hincapié en la Mediación familiar; posteriormente, describiremos el perfil de mediador desde su marco legal en España para complementarlo con los aspectos académicos que lo estructuran. Una vez realizado lo anterior, se dará una breve introducción al concepto de IM de Howard Gardner definiendo las 4 (Lógico-matemática, Lingüística, Intrapersonal e Interpersonal) de las 7 inteligencias (el resto son la Cinestésico-corporal, Musical y Espacial) que se consideraron para la aplicación del instrumento y los resultados obtenidos del mismo al aplicarse a un grupo de profesionales que ejercen la mediación familiar intrajudicial en un juzgado del sur de España y, finalmente, presentar una estructuración de las competencias del mediador desde el marco de las IM.

Se desarrolló una entrevista semiestructurada compuesta por 13 preguntas con la finalidad de explorar la percepción de profesionales responsables de centros y de profesionales que ejercen como mediadores familiares con la finalidad de obtener de ellos "los factores intrínsecos... que los hacen ser, pensar o sentir..." (Sáenz \& Téllez-Castilla, 2014, pág. 172) con respecto a la ejercicio profesional y la eficacia en el procedimiento de mediación.

Se optó por este tipo de técnica ya que lo que se busca es comprender la opinión de las personas de las cuáles consideramos como expertos en el ejercicio de la profesión del mediador familiar, poder categorizar sus respuestas para poder explicar, desde el marco de las inteligencias múltiples con la finalidad explorar la posibilidad de impulsar la profesionalización del mediador (Sáenz \& Téllez-Castilla, 2014, pág. 172).

A través de una codificación de las variables, se desarrolló un guion de preguntas para explorar la cómo los facilitadores de mediación con la intención de realizar un análisis desde el marco de inteligencias múltiples, lo cual permite una nueva perspectiva de la profesionalización de la mediación.

Para llevar a cabo la aplicación se estableció contacto con la persona responsable de la unidad de mediación intrajudicial en materia familiar de un centro al sureste de España, se explicó la propuesta de investigación y se solicitó permiso para realizar la aplicación 
correspondiente a la cual accedieron amablemente y en la cual participaron 11 profesionales que ejercen como mediadores familiares y la responsable de la unidad. La muestra estaba compuesta por profesionales entre 1 y 16 años de experiencia en mediación familiar de los cuales el 70\% son egresados del campo del Derecho y el resto de los campos de Psicología y Ciencias del trabajo, el $70 \%$ de la muestra es de género femenino.

En la etapa posterior a la aplicación del instrumento cualitativo se prosiguió a realizar la transcripción de las grabaciones de las entrevistas para importarlas al programa de análisis cualitativo MAXQDA para Windows, software por medio del cual se realizó el análisis de las respuestas obteniendo los siguientes resultados.

\section{LAS INTELIGENCIAS MÚLTIPLES}

A partir de lo postulado por Thurstone en 1960 y Guilford en 1967, y retando las teorías existentes hasta le fecha en relación a la inteligencia, Gardner propone la existencia de siete tipos de inteligencia a las cuales denominó Inteligencias Múltiples (IM) (2001, pág. 5).

La propuesta de Gardner se plantea una nueva definición para la inteligencia, opuesta la idea del Coeficiente Intelectual (CI, o IQ en inglés) que hace referencia a la este concepto como la calificación obtenida en una prueba de lápiz y papel que tiene como fin el proyectar el desempeño académico de una persona en el ambiente escolar; contrario a esto, la teoría de inteligencias múltiples hace referencia a la inteligencia como "la capacidad de resolver problemas, o de crear productos, que sean valiosos en uno o más ambientes culturales." (Gardner, 2001, pág. 5).

La idea central de Gardner es que considera que una competencia intelectual humana no haría referencia a una calificación de una evaluación, sino que "debe dominar un conjunto de habilidades para la solución de problemas" (2001, pág. 60) con la finalidad de que el individuo pueda encontrar soluciones genuinas a las dificultades en las que se encuentre, incluso si esto implica la creación de un producto. Es por esta razón que se considera apropiado el uso de este marco para evaluar el ejercicio profesional de mediación familiar, ya que no es a través de una 
evaluación el cómo se puede evaluar el desempeño, sino en la capacidad para procurar las condiciones en medio de un conflicto con la finalidad de dirigirlo a una solución constructiva que se vea plasmada en un acuerdo de mediación.

Para efectos de simplificar el contenido del presente documento se definirán únicamente las inteligencias que son pertinentes a la investigación Macías (2002, págs. 34-35) propone las siguientes definiciones:

- Inteligencia lingüística: "Se refiere a la adecuada construcción de oraciones, la utilización de palabras de acuerdo a su significados y sonidos, al igual que la utilización del lenguaje de conformidad con sus diversos usos."

- Inteligencia lógico-matemática: "Referida a la facilidad para manejar cadenas de razonamiento e identificar patrones de funcionamiento en la resolución de problemas."

- Inteligencia Intrapersonal: "Señala la capacidad que tiene una persona para conocer su mundo interno, es decir, sus propias y más íntimas emociones y sentimientos, así como sus propias fortalezas y debilidades."

- Inteligencia Interpersonal: "Muestra la habilidad para reconocer las emociones y sentimientos derivados de las relaciones entre las personas y sus grupos."

La manera en cómo se ancla esta definición de inteligencia en la mediación es que el mediador tiene la función de ayudar a las partes en conflicto (capacidad de resolver problemas) a generar un acuerdo (generar productos) a través de la mutua voluntad de los participantes que tendrá un efecto procesal, al disminuir las cargas en los juzgados, y uno social, al facilitar la "impetración de la justicia” (Gorjón, 2015) y tener un efecto constructivo en el tejido social (valor cultural).

Desde la impetración de la justicia, se considera el capital humano como uno de los pilares para la implementación, desarrollo y evolución de los métodos alternos de solución de conflictos pues considera que:

"Las bases de estos métodos son las habilidades, los conocimientos, los valores y las aptitudes de los negociadores, de los mediadores y de los árbitros, así como la ciudadanía en general cuando interviene en un proceso de este tipo, estas competencias sustentan el capital intelectual, que a su vez se integra por el capital humano, el capital estructural y el capital relacional como una de las formas más comunes de agruparlos." (Gorjón, 2015, pág. 125). 
Es entonces que podemos ver que la exploración del fenómeno de los métodos alternos de solución de conflictos desde ciencias y paradigmas multidisciplinarios aportará conocimiento que permita el desarrollo de la ciencia.

El considerar el marco de las inteligencias múltiples para clasificar las competencias en la mediación puede ser un paso hacia delante en la idea de evaluar la calidad y los resultados del ejercicio profesional de la mediación, se considera la mediación familiar como el pionero para la aplicación de esta metodología por la relevancia que tiene para la sociedad, así como por ser el ámbito de aplicación donde es necesario mejorar los indicadores de generación de acuerdos ya que, de acuerdo a resultados de la propia unidad de mediación familiar, no supera el $50 \%$ de generación de acuerdos en relación a los expedientes que inician el proceso de mediación.

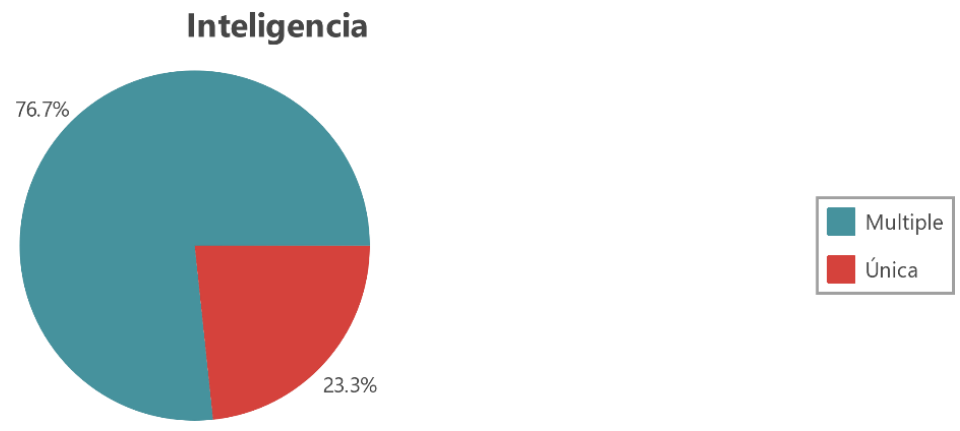

Concepto de Inteligencia para mediadores

En relación a lo anterior, cuando se les cuestionó a los profesionales de mediación respecto al concepto de la inteligencia; la mayoría, sin utilizar términos técnicos como los desarrollados en la teoría de Gardner, concordaba con la conceptualización de una inteligencia múltiple en lugar de considerarla como un constructo único.

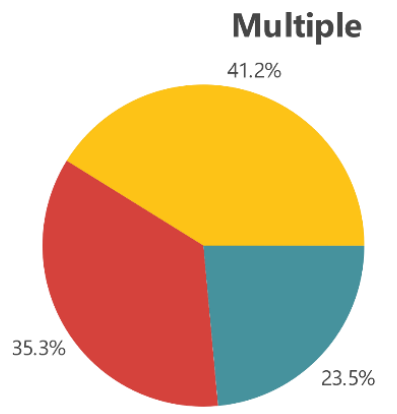


Esta inteligencia múltiple descrita por los mediadores incluye, además de lo que se considera como la inteligencia académica la cual se refiere a la capacidad de obtener buenos resultados o contar con conocimientos meramente académicos, capacidades y habilidades que hacen referencia a considerar los diferentes factores que inciden en un fenómeno, la capacidad de comprenderlos y analizarlos de una manera creativa o que sea "fuera de la caja", refiriéndose a ver soluciones que probablemente no se hayan contemplado con anterioridad, con la finalidad de aportar soluciones a dicho fenómeno que sean las más apropiadas, considerando que se el ser humano busca la supervivencia en un medio natural, el cual incluye el aspecto social del mismo, que no se puede dejar de lado.

\section{DEFINICIÓN DE MEDIACIÓN EM EL ÁMBITO FAMILIAR}

El documento de las Directrices de las Naciones Unidas para una mediación eficaz, donde se describe la mediación como "un proceso por el que un tercero ayuda a dos o más partes, con su consentimiento, a prevenir, gestionar o resolver un conflicto ayudándolos a alcanzar acuerdos mutuamente aceptables” (2012, pág. 4). En España, La Ley 5/2012 define a la mediación como "aquel medio de solución de controversias, cualquiera que sea su denominación, en que dos o más partes intentan voluntariamente alcanzar por sí mismas un acuerdo con la intervención de un mediador.” (2012, pág. 7)

Sáenz y Rivera citan a Folberg \& Taylor (1992) en su obra La mediación, concepto y ámbitos de aplicación (2012, pág. 201), donde se refiere a la mediación como:

\footnotetext{
“el proceso mediante el cual los participantes, junto con la asistencia de una persona o personas neutrales, aíslan sistemáticamente los problemas en disputa con el objeto de encontrar opciones, considerar alternativas, y llegar a un acuerdo mutuo que se ajuste a sus necesidades. La mediación es un proceso que hace hincapié en la propia responsabilidad de los participantes de tomar decisiones que influyen en sus vidas"
}

Ambos conceptos atienden, de cierta manera, lo propuesto por las Naciones Unidas al considerar a la mediación como la herramienta o vía alternativa de solución de conflictos que se pueda presentar entre dos o más partes; sin embargo, el término "proceso" se consideraría el más adecuado ya que dentro de un proceso pueden existir mecanismo o medios para llevarlo a 
cabo. De esta manera definiríamos la mediación como un proceso al cual dos o más partes en conflicto, que no han podido encontrar una solución al mismo, solicitan el apoyo de una tercera parte, imparcial al conflicto, de manera voluntaria para que les guíe en el proceso de buscar una solución que atienda de manera equitativa sus intereses y necesidades.

Con lo que respecta a la mediación familiar específicamente, no sería muy diferente al proceso anteriormente descrito; por el mismo concepto de "familiar" se sobrentiende que los involucrados en el conflicto son personas que pertenecen a un mismo grupo social denominado familia y que comparten un vínculo que puede oscilar en dos aspectos altamente complejos; un vínculo social, con un marco normativo que le atiende dentro de la mediación, y otro vínculo emocional-psicológico, que también tiene un efecto para el procedimiento de mediación, sin embargo no desde un aspecto normativo, sino como un elemento que le distingue de los demás ámbitos de la mediación; ya que, el factor humano se vuelve particularmente determinante en este tipo de proceso, lo cual exige un papel puntal por parte del mediador (Sáenz López \& Rivera Hernandez, 2012).

Lo anterior lo describe también De Villa al describir que en los conflictos familiares que se presentan en mediación "es común que los sentimientos y la falta de comunicación profundicen un problema.” (De Villa Cortés, 2012, pág. 20). Es aquí el interés del presente artículo, se tiene en claro que el mediador no hace un trabajo terapéutico con los participantes de la mediación familiar pues se tiene en claro que "la mediación estructuralmente considerada no persigue prima facie la mejora de las relaciones sino la resolución del conflicto" (Serrano, 2008, pág. 55); sin embargo, el mediador debe contar con habilidades y capacidades para identificar, comprender y gestionar efectivamente el elemento emocional- afectivo que se presente en la mediación. El marco de las Inteligencias Múltiples puede ser una estructura que permite comprender de una manera más sencilla este aspecto para el profesional de mediación. 


\section{PERFIL DO MEDIADOR}

Iglesias y Pastor consideran que la calidad y la eficacia del ejercicio profesional de la mediación está directamente en función de la formación profesional recibida y laconsideran como una variable fundamental para el resultado de la mediación (Iglesias-Ortuño \& PastorSeller, 2015).

Por su parte, Serrano, a través de estudios enfocados al desempeño de los profesionales de mediación en los cuales consideró las respuestas de los usuarios a los procesos, concluyó que las "habilidades sociales" tenían una incidencia mayor en el resultado del proceso de mediación que la formación de los profesionales, de lo anterior da dos explicaciones posibles a estas conclusiones: 1)"Puede deberse a que el sujeto que pasa por la mediación la da por supuesto y por consiguiente no la percibe de manera relevante" y 2) la posibilidad de que "son las habilidades sociales mencionadas las que en mayor medida posibilitan un clima y unas condiciones para afrontar el conflicto constructivamente" (Serrano, 2008, pág. 56).

Al buscar poner ambos lados en sintonía consideramos que ambos aspectos tienen una incidencia relevante al momento de ejercer el rol de mediador; la formación profesional obtenida facilita la comprensión y estructuración del conflicto, los pasos dentro del proceso de mediación, las herramientas y técnicas a utilizar, y la estrategia de gestión de todo lo anterior (que en caso de las IM haríamos referencia a las Inteligencias Lógico-matemática y Lingüística), mientras que las habilidades sociales (Inteligencias Intrapersonal e Interpersonal) permitirían la aplicación efectiva de los principios de la mediación por parte del profesional que se presenta como tercero imparcial (Imparcialidad, Neutralidad, Objetividad, Honestidad, etc.), así como propiciar un ambiente de confianza y de comunicación efectiva entre las partes para una solución constructiva del conflicto.

Al relacionar el concepto de inteligencia de manera específica con el ejercicio de la mediación no hay mucha con lo descrito en los párrafos anteriores. Al explicar qué es un mediador inteligente, los profesionales en el campo coinciden que es una persona que debe tener la capacidad de identificar aspectos críticos dentro de los conflictos, comprender los factores y actores que se encuentran involucrados en los mismos, comprender cómo se 
comportan estos factores y actores, y analizar la manera en la cual debe procurar la interacción con ellos y entre ellos, con la finalidad de alcanzar soluciones que probablemente no habían aparecido en su horizonte y así alcanzar acuerdos.

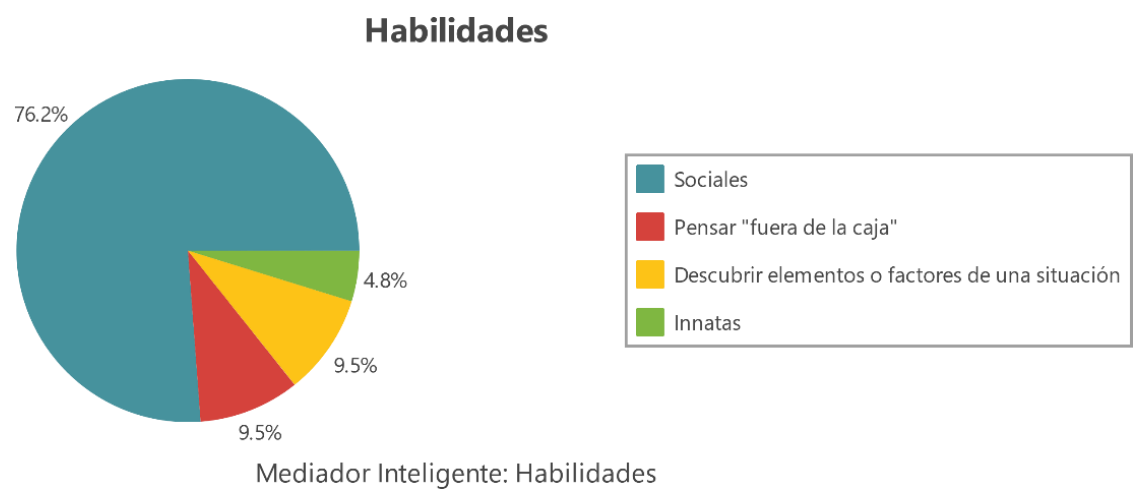

\section{ELEMENTOS LEGALES}

Dentro de la Ley 5/2012, de 6 de julio, de Mediación en Asuntos Civiles y Mercantiles, Ley que actualmente está vigente y que atiende la materia de Mediación Familiar en España , en el apartado número III del Preámbulo hace menciona de un aspecto relevante en referencia al mediador ya que, aunque se entiende que el éxito del proceso de mediación no depende del mediador, considera a éste como "una pieza esencial del modelo, pues es quien ayuda a encontrar una solución dialogada y voluntariamente querida por las partes." (Jefatura del Estado (España), 2012, pág. 5).

Así mismo, explica que la actividad profesional de la mediación "se despliega en múltiples ámbitos profesionales y sociales, requiriendo habilidades que en muchos casos dependen de la propia naturaleza del conflicto." (Jefatura del Estado (España), 2012, pág. 5). Exigiéndole al mediador una formación pertinente que le permita desarrollar su ejercicio profesional ofreciendo garantía a las partes que participan del proceso (Jefatura del Estado (España), 2012). En el caso de la mediación familiar el conocimiento, identificación, 
comprensión y gestión de los aspectos afectivo-emocionales se vuelve un elemento trascendental al momento de gestionar conflictos.

Posteriormente, dentro del "Título III Estatuto del mediador" de la Ley 5/2012 se encuentra el "Art. 11. Condiciones para ejercer de mediador", compuesto por 3 sub apartados que hacen énfasis a: 1) Los derechos civiles y la habilitación legal del ejercicio profesional, así como cumplir con los requisitos necesarios, 2) Documentos probatorios que habilitan a una persona para ejercer como mediador y 3) La responsabilidad civil con las partes que solicitan su servicio.

Dentro de su Art. 13 de la "Actuación del mediador" establece los criterios para que el facilitador de mediación ejecute su función, compuesto por 5 subapartados y dos párrafos, describe lo siguiente:

1. El mediador facilitará la comunicación entre las partes y velará porque dispongan de la información y el asesoramiento suficientes.

2. El mediador desarrollará una conducta activa tendente a lograr el acercamiento entre las partes, con respeto a los principios recogidos en esta Ley.

3. El mediador podrá renunciar a desarrollar la mediación, con obligación de entregar un acta a las partes en la que conste su renuncia.

4. El mediador no podrá iniciar o deberá abandonar la mediación cuando concurran circunstancias que afecten a su imparcialidad.

5. Antes de iniciar o de continuar su tarea, el mediador deberá revelar cualquier circunstancia que pueda afectar a su imparcialidad o bien generar un conflicto de intereses. Tales circunstancias incluirán, en todo caso:

a) Todo tipo de relación personal, contractual o empresarial con una de las partes.

b) Cualquier interés directo o indirecto en el resultado de la mediación.

c) Que el mediador, o un miembro de su empresa u organización, hayan actuado anteriormente a favor de una o varias de las partes en cualquier circunstancia, con excepción de la mediación. 
I. En tales casos el mediador sólo podrá aceptar o continuar la mediación cuando asegure poder mediar con total imparcialidad y siempre que las partes lo consientan y lo hagan constar expresamente.

II. El deber de revelar esta información permanece a lo largo de todo el procedimiento de mediación.

En lo que corresponde a la cantidad de horas de formación mínima para la mediación, de acuerdo con Iglesias-Ortuño, en España hace la observación de que, aunque en lo competente a la formación práctica de la formación específica en mediación las leyes autonómicas y estatales tienen determinado precisamente la cantidad a cumplir, en lo correspondiente a la formación específica existe una diferencia importante en cuanto a la cantidad de horas a cumplir para ser reconocido como mediador. Mientras que las normas autonómicas exigen un mínimo de 200 horas en la que más de la mitad de ellas tienen que estar relacionadas a una titulación universitaria, las normas estatales establecen un mínimo de 100 horas; además que, la normativa se actualizado en el sentido que "la formación ya no se circunscribe únicamente al ámbito familiar, sino que se extiende a los diferentes ámbitos de lo civil, mercantil y social debido a una paulatina proliferación de ámbitos...”(Igleias-Ortuño, 2016, pág. 148)

\section{ELEMENTOS ACADÉMICOS}

El modelo por competencias es el que los sistemas educativos profesionales han seleccionado como el más apropiado a la hora de formar a sus educandos ya que considera los aspectos más actuales en relación con el aprendizaje y la educación, este modelo tiene como punto vertebral ir más allá que obtener el aprendizaje a través del conocimiento, busca el desarrollo del alumno a través de la comprensión de conceptos, el dominio de los contenidos y propiciar una autonomía para la aplicación de éstos, así como el impacto y los resultados de esta aplicación de una manera crítica en una materia específica en la que se profesionaliza (Iglesias-Ortuño \& Pastor-Seller, 2015, pág. 53). 
Utilizando el fundamento de las competencias; la idea es enmarcar las competencias del perfil de mediación, que como hemos observado requiere no solo de un desarrollo y dominio dentro de una materia específica, sino que también requiere de un conjunto de habilidades y capacidades personales que tienen un impacto relevante en el proceso de mediación. El ejercicio de mediador requiere características técnicas y humanas, puesto que "el mediador ha de fomentar una negociación basada en el diálogo y la comunicación eficaz que permita a las partes tratar problemas relevantes" (Igleias-Ortuño, 2016, pág. 150) y es el encargado de propiciar un espacio, a través de sus conocimientos en el conflicto y los métodos alternos, para transformar la perspectiva de los participantes hacia posiciones más flexibles hacia una solución, como lo menciona Iglesias-Ortuño: "así mismo el mediador debe proveer a las partes de estrategias y habilidades que conformen un aprendizaje para la resolución de futuras situaciones de conflicto." (2016, pág. 150)

Gorjón y Steele describen las competencias de los prestadores de los servicios de los métodos alternos de solución de conflictos en generales y específicas. Las primeras van relacionadas con el "conocer, analizar, aplicar, diseñar e innovar" (2012, pág. 24) las técnicas que se utilizan para la resolución de conflictos, así como las habilidades necesarias dentro de sus ámbitos de influencia y, de manera interesante, menciona el encaminar estas estrategias a través de un conocimiento transversal de teorías, conceptos y técnicas que permitan atender puntualmente las circunstancias del conflicto "en que se encuentren presentes temores, sentimientos agresivos o conductas inadecuadas de los contendientes” (2012, pág. 24).

Al referirse a las competencias específicas de los prestadores de servicio de los métodos alternos, Gorjón y Steele las enfocan a la ejecución del rapport, que tiene una vital relevancia en las interacciones interpersonales y que tiene efecto en la capacidad de reestructurar la comunicación a través de diferentes técnicas que se pueden utilizar, así como el conocimiento y aplicación de técnicas de control de ira durante el proceso, haciendo hincapié en el aspecto emocional en el que se ve envuelto los procesos de mediación (2012, pág. 25).

Como hemos descrito anteriormente, la aplicación del instrumento a profesionales prestadores de servicio de mediación familiar permitió poner a la luz que tanto la formación profesional (la obtención de conocimientos, estrategias, herramientas y técnicas) tienen un peso importante al momento de la gestión de los conflictos, de acuerdo al desarrollo teórico de la 
mediación ( (Iglesias-Ortuño \& Pastor-Seller, 2015) e (Igleias-Ortuño, 2016)), y que, a su vez, las "habilidades sociales", desde la perspectiva de los usuarios, son las que permiten que su participación fluya de una manera cooperativa con el mediador, lo que por ende acerca el conflicto a una solución constructiva a través de la voluntariedad (Serrano, 2008)“

Aunque existe la opinión dividida a cerca de que, si la formación en mediación debería ser en posgrado, posterior a la finalización de estudios universitarios en campos afines a la materia o que esta formación podría darse ya como una profesión universitaria concreta que "permitirá adquirir las nociones básicas de esta disciplina y posteriormente ampliar, desarrollar y aplicar en la fase del posgrado.” (Iglesias-Ortuño \& Pastor-Seller, 2015, pág. 88). La perspectiva propuesta podría fortalecer las conclusiones de Iglesias-Ortuño y Pastor- Seller de que se puede "concebir la mediación como una profesión que ha de aprenderse mediante una formación basada en los parámetros del Espacio Europeo de Educación Superior y que ha de estar fundamentada en el aprendizaje por competencias." (Iglesias- Ortuño \& Pastor-Seller, 2015, pág. 88)

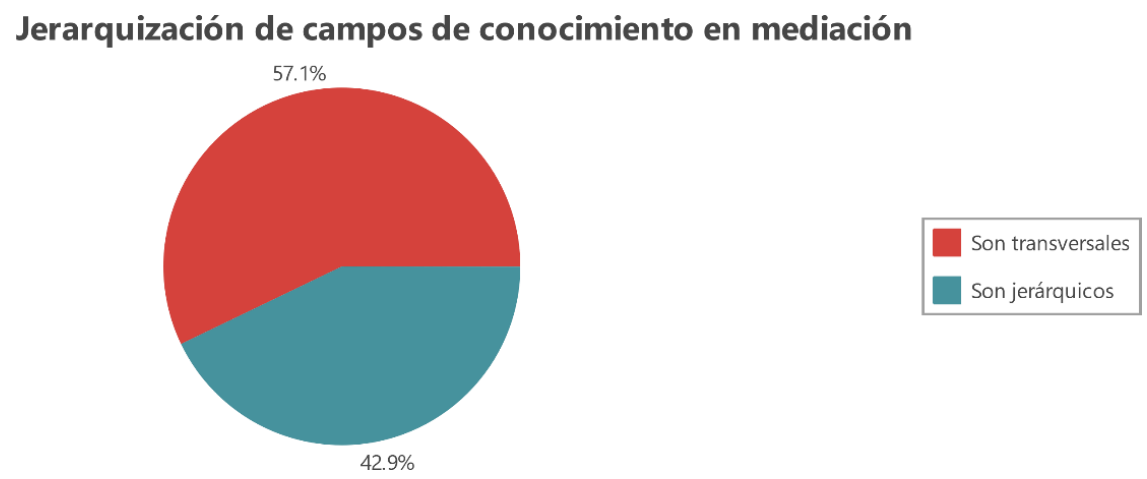

Lo descrito en párrafos anteriores se sustenta en los resultados obtenidos en las entrevistas a los facilitadores confirman lo descrito en el párrafo anterior, ya que los mismos profesionales consideran que la formación exclusiva en mediación no es suficiente. Explicaban que el Derecho aporta, principalmente, el marco jurídico que debe saber el mediador para poder dirigir el proceso hacia acuerdos que se encuentren dentro de la ley; la Psicología aporta, relevantemente, información acerca de las técnicas de rapport y de gestión emocional, tanto de manera personal como para la gestión de las emociones de los participantes del procedimiento. 
Campos profesionalizantes de mediación

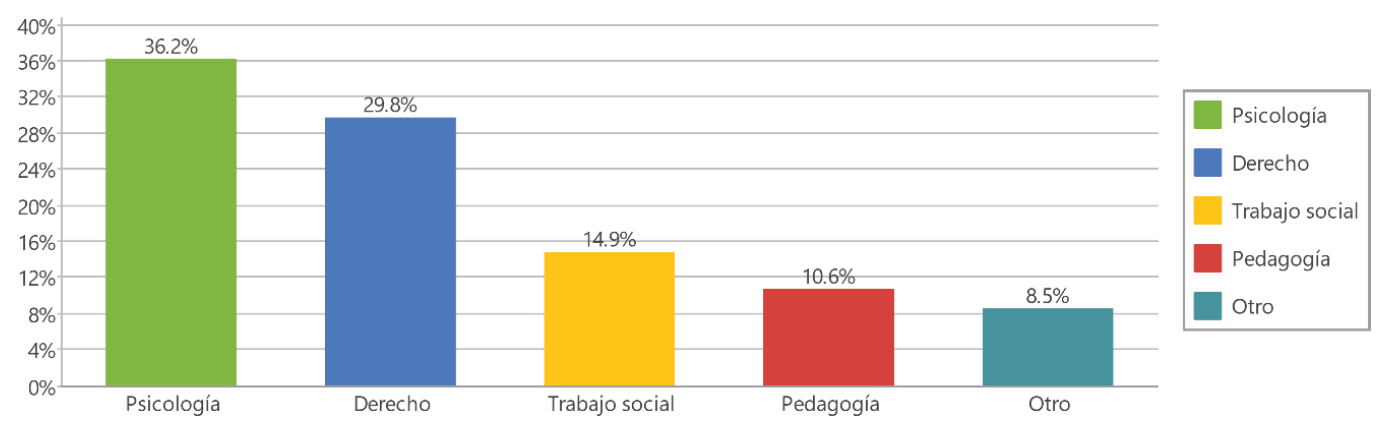

\section{EL PERFIL DEL MEDIADOR EM EL MARCO DE LAS INTELIGENCIAS MÚLTIPLES}

En un principio aclaramos que en el instrumento no se consideró la inteligencia Lógicomatemática puesto que se considera en estrecha relación con los pasos del proceso de mediación; como se pudo exponer anteriormente el mediador no puede ejercer el ejercicioprofesional si no tiene la preparación pertinente a los métodos alternos de solución de conflictos.

De la información más relevante con respecto a las inteligencias múltiples en el ejercicio de la mediación es que, de las tres inteligencias evaluadas en las cuales dos se relacionan estrechamente con las competencias establecidas para el mediador, el tema en el cual los entrevistados ahondaron a la hora de mencionar sus respuestas fue en referencia a la inteligencia intrapersonal del mediador.

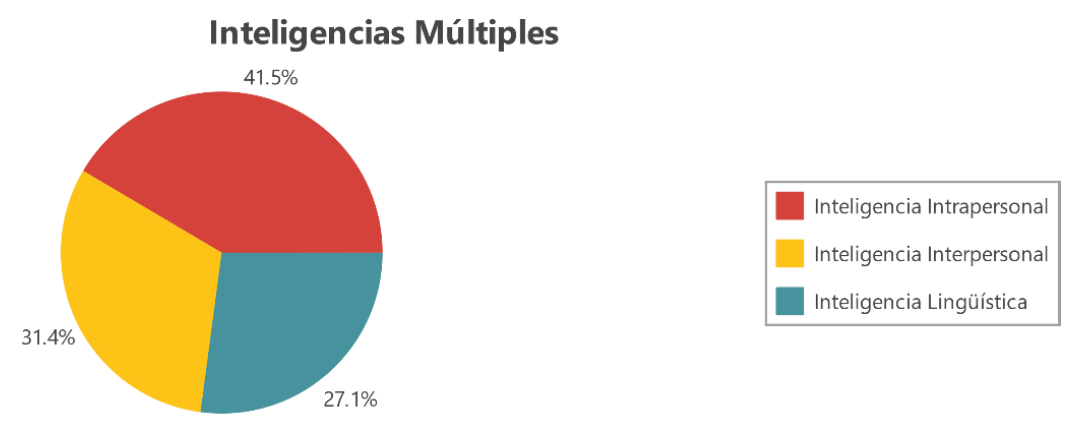

Aportes de entrevistados en el marco de IM 
Para la investigación nos dimos a la tarea de identificar en la actualidad las competencias establecidas para la formación del profesional en mediación y encontramos que, al clasificarlas dentro de un marco interpretativo a partir de la teoría de las inteligencias múltiples, la Inteligencia Intrapersonal no cuenta con una distribución equitativa en relación a las competencias que se relacionan con las Inteligencias Lógico-matemática, Lingüística e Interpersonal como podemos ver a continuación al considerar lo propuesto por Gorjón y Sánchez (2015), así como por Iglesias-Ortuño y Pastor-Seller (2015).

\begin{tabular}{|c|c|c|c|c|}
\hline Fuente & Lógico-matemática & Lingüistica & Intrapersonal & \begin{tabular}{|l|} 
Interpersonal \\
\end{tabular} \\
\hline 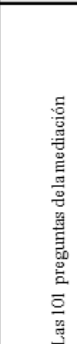 & $\begin{array}{l}\text { II. Conoce los diferentes modelos de me diacióny sus } \\
\text { técnicas. } \\
\text { III. Distingue entre los diferentes modelos de mediación } \\
\text { y/o conciliación } \\
\text { IV. Conoce las técricas dela me diacióny de la } \\
\text { conciliación. } \\
\text { XI. Estructura el plan de me diación y conciliación } \\
\text { XII. Conoce las distintas etapasprocedimentales desdeel } \\
\text { plan-tratamiento del conflicto hasta el acuerdo. } \\
\text { XVI. Definen y distinguenlas técnicas, teoriasy conceptos } \\
\text { asociados de control de crisis en unconflicto. } \\
\text { XVIII. Concretanlas opciones que favorezcana las partes, } \\
\text { que sean susceptibles de conveniry yue no alteren el orden } \\
\text { público. }\end{array}$ & $\begin{array}{l}\text { V. Son capaces de reestructurar la } \\
\text { comunicación delas partes a través de } \\
\text { técnicas de comunicación } \\
\text { VI. Desarrolla el sentido dela escucha } \\
\text { activa. } \\
\text { VII. Desarrolla la técnica del parafraseo. } \\
\text { XIII. Son capaces de comprender la } \\
\text { historia del conflicto para detectar si es } \\
\text { susceptible de iniciarl la me diación o la } \\
\text { conciliación } \\
\text { XX. Elaboran el acuerdo o converio en el } \\
\text { que las partes secercioran de que sus } \\
\text { pretensiones han sido satisfechas. }\end{array}$ & & $\begin{array}{l}\text { I. Conoce e identifica las materias que son } \\
\text { susceptibles de mediar o conciliar. } \\
\text { VIII. Conoce y aplica la técrica del Caucus como } \\
\text { elemento sine qua non dela me diación conciliación. } \\
\text { IX. Conoce y aplica la técrica de control de la ira } \\
\text { durante el procedimiento de me diación-concliación. } \\
\text { X. Conoce y aplica la técnica del rapport. } \\
\text { XIV. Detecta las posiciones de las partes en el } \\
\text { conflicto. } \\
\text { XV. Detecta los intereses de las partes. } \\
\text { XVII. Son capaces de motivar opciones para la } \\
\text { solucióndel conflicto por parte de los involucrados. } \\
\text { XIX. Son capaces de sustraer los interesesy } \\
\text { necesidades mutuas delos protagonistas del conflicto }\end{array}$ \\
\hline 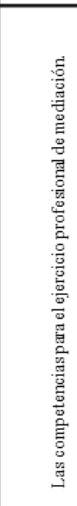 & $\begin{array}{l}\text {-Transversales: } \\
\text { Ciencias sociales } \\
\text { Ciencias juridicas } \\
\text { Sociologia } \\
\text { Psicologia } \\
\text { Pedagogia } \\
\text { Investigación social } \\
\text { - Generales: } \\
\text { Comprensión del beneficio de la colaboración de las } \\
\text { diferentes áreas del conocimiento } \\
\text { Dominio de la planificación } \\
\text { Comprensión, análisis, critica y estudio } \\
\text { Adaptación de la teoria a la práctica } \\
\text { - Específicas: } \\
\text { Dominio del conflicto } \\
\text { Capacidad para desarrollar el proceso de me diación, sus } \\
\text { fases y protocolos de actuación } \\
\text { Desempeño del rol de mediador } \\
\text { Distinción de roles respecto a otras disciplinas } \\
\text { Respeto al código ético reconocido } \\
\text { - Individuales: } \\
\text { Consideraciónde la formación contirua }\end{array}$ & $\begin{array}{l}\text { - Generales: } \\
\text { Conocimiento de las variables de la } \\
\text { comunicación } \\
\text { - Específicas: } \\
\text { Dominio del conflicto } \\
\text { Ejecución de las habilidades y técricas } \\
\text { delmediador (escucha activa, } \\
\text { comunicación eficaz) } \\
\text { Desempeño del rol de mediador }\end{array}$ & $\begin{array}{l}\text { - Específicas: } \\
\text { Ejecución de las habilidadesy } \\
\text { técnicas del mediador } \\
\text { (imparcialidad neutralidad) } \\
\text { Desempeño del rol de mediador } \\
\text { - Individuales: } \\
\text { Ejecutarun talante adecuado } \\
\text { como guia del proceso }\end{array}$ & $\begin{array}{l}\text { - Específicas: } \\
\text { Dominio del conflicto } \\
\text { Desempeño del rol de mediador } \\
\text { - Individuales: } \\
\text { Promoción de la confianza en las partes } \\
\text { Eficacia en las relaciones personales }\end{array}$ \\
\hline
\end{tabular}

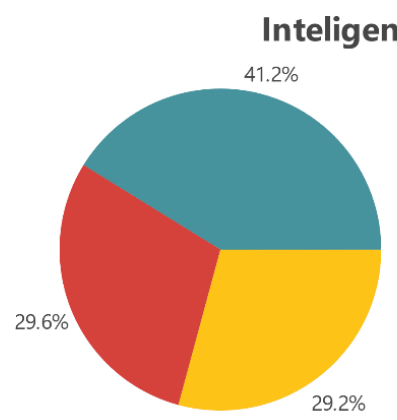

Amplitud y aplicación del léxico

Regulación de volumen y tono de voz

Asertividad en la comunicación

Elementos de la I-Lingüística presentes en la mediación 


\section{La inteligencia Lingüística}

Los resultados con respecto a la inteligencia lingüística en el ejercicio de mediación fortalecen las propuestas de que la comunicación es la herramienta por excelencia en el procedimiento de mediación; el contar con un léxico amplio, el utilizar conscientemente su volumen y tono de voz para influir en los temperamentos y estados de ánimo de los participantes para propiciar una comunicación empática entre ellos y captar de manera efectiva su atención, así como el procurar la asertividad en sus intervenciones permite hacer demostrable a las partes los principios de imparcialidad, neutralidad y objetividad que se postulan para la mediación, puesto que es una manera de comunicación que permite expresar objetivamente los elementos del conflicto y permite influencia a las partes a que haya un dialogo constructivo entre ellos al señalar que la comunicación debe ser respetuosa.

\section{La inteligencia Interpersonal}

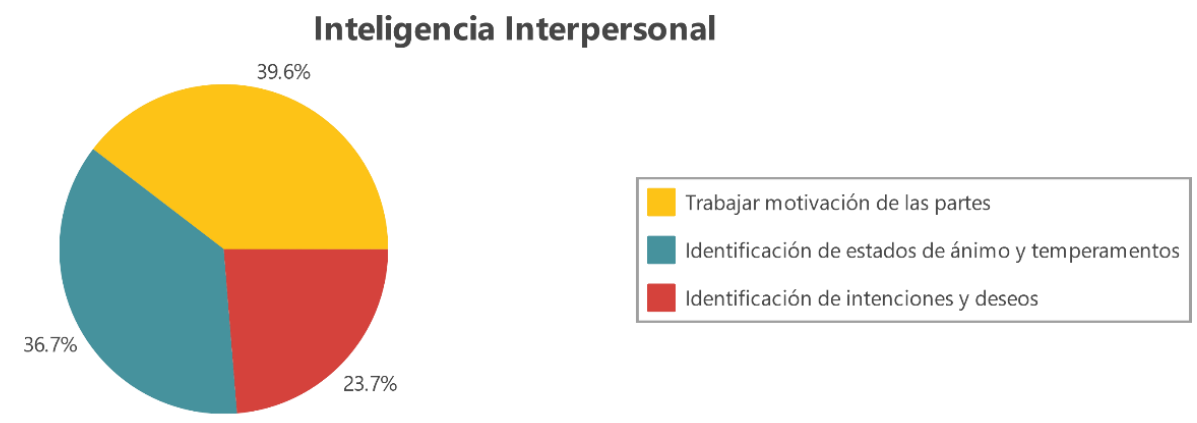

Elementos de la I-Interpersonal presentes en la mediación 
A través de la adecuada aplicación de la comunicación en el procedimiento de mediación, el mediador puede utilizar sus conocimientos, habilidades y capacidades relacionadas con la inteligencia interpersonal para transformar la motivación de las partes en vísperas de lograr soluciones al conflicto que se enfrentan; a través de la inteligencia interpersonal el mediador es capaz de identificar los intereses en común y los intereses más polarizados en los cuales el mediador tiene la responsabilidad de procurar un ambiente de flexibilidad y creatividad para que las partes busquen un terreno en común con la finalidad potencializar la motivación de las partes a la búsqueda de soluciones en conjunto.

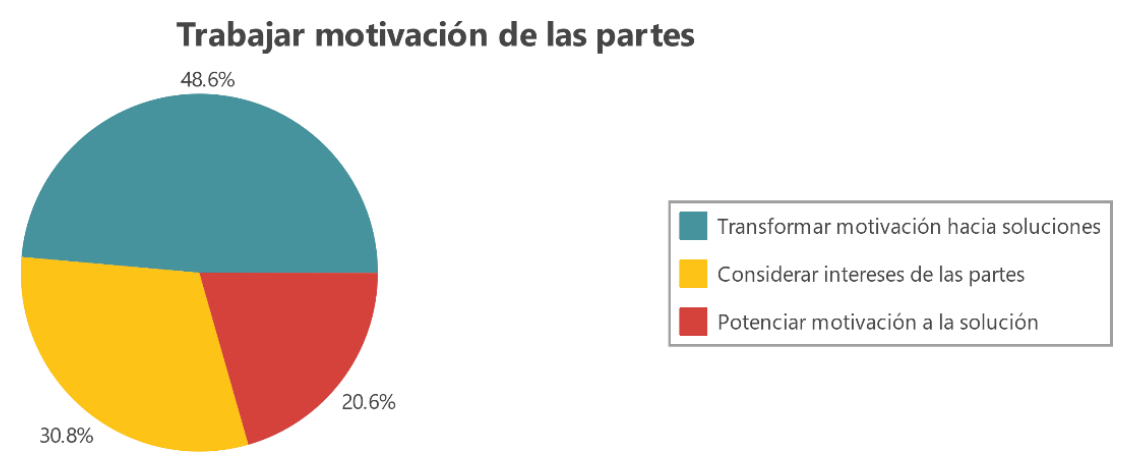

Para lo anterior es necesario que el mediador aplique a conciencia plena la escucha activa con el fin de analizar el discurso de las partes e identificar, analizar y comprender en sus expresiones, a través de los elementos verbales y no verbales, los aspectos emocionales y los elementos objetivos del conflicto, así como las condiciones en las que se encuentra el vínculo entre las partes en el momento del conflicto.

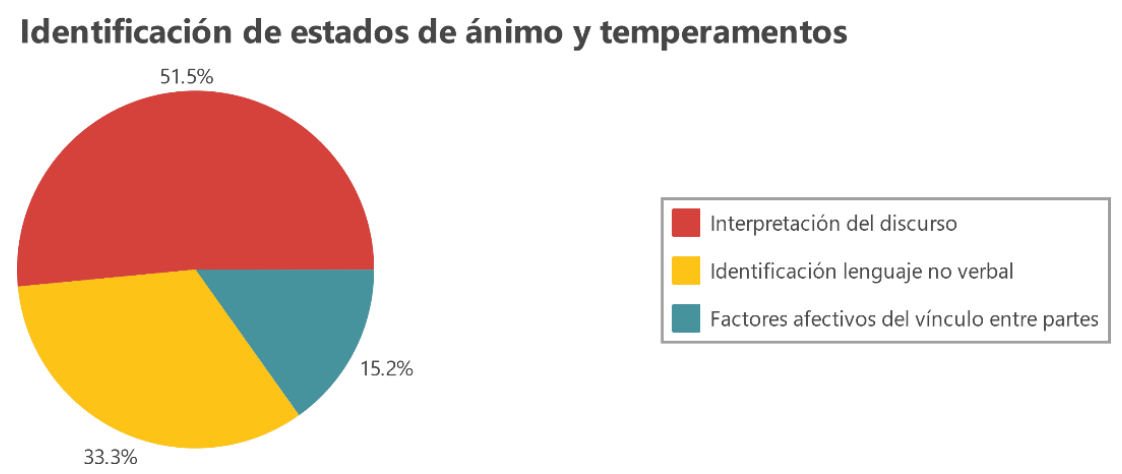




\section{Inteligencia intrapersonal}

Con lo que respecta a la inteligencia intrapersonal, los hallazgos obtenidos de las entrevistas resuenan en los principios establecidos para el procedimiento de mediación y para el ejercicio de la mediación, la imparcialidad, la neutralidad y la objetividad; además que forman un elemento vertebral para la capacidad de la gestión emocional aspecto puntal de la mediación familiar.

Esto lo podemos ver en que, de acuerdo a las respuestas de los entrevistados, los mediadores tienen que ser capaces de canalizar sus emociones para llegar a una regulación de la conducta verbal como no verbal en el ejercicio de la mediación, pues es la manera de hacer visible la imparcialidad, neutralidad y objetividad del ejercicio profesional.

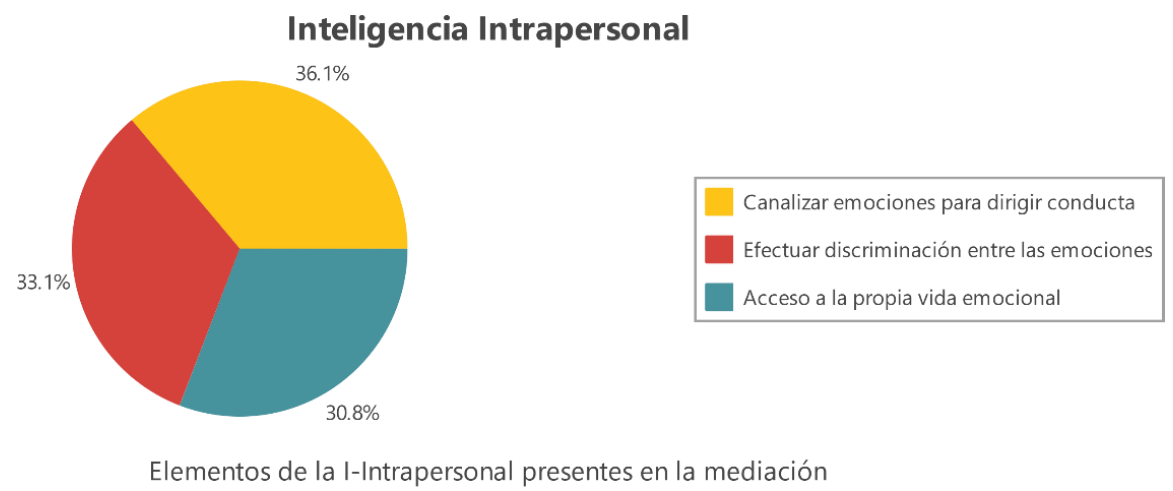

Para lograr esta canalización de la conducta es indispensable lograr una conciencia emocional antes, durante e incluso posterior al ejercicio de la mediación. Lo que permite esta conciencia emocional es contar con un sistema simbólico de las emociones conocidas y reconocidas que permita identificar el estado emocional en el que se encuentra el mediador y si este estado es favorable para llevar a cabo el ejercicio profesional. 


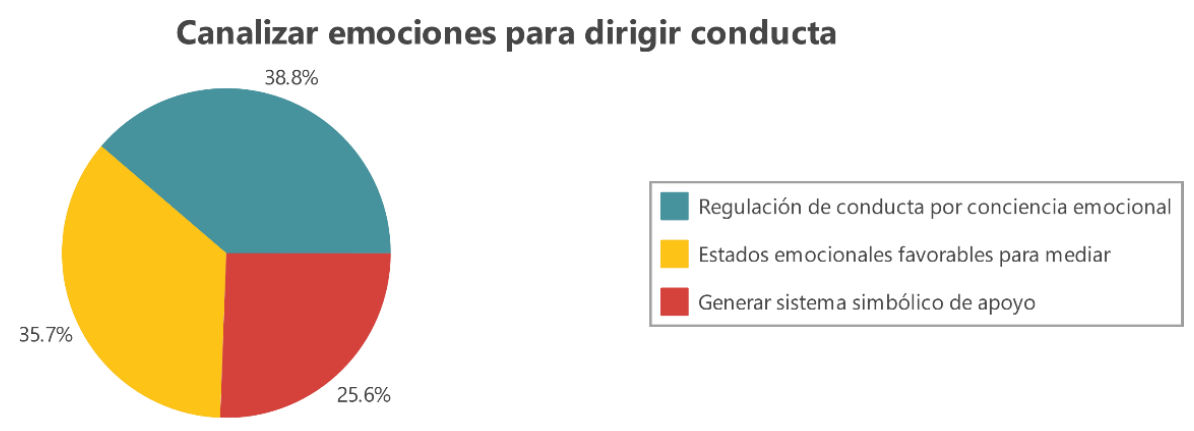

Canalizar las emociones para dirigir la conducta

Esta regulación de la conducta con el apoyo del sistema simbólico, de acuerdo a las respuestas de los entrevistados, se logra gracias a un control de los estados cognitivos que son provocados por las emociones experimentadas, pues se tiene una conciencia de los efectos que generan las emociones. Todo lo anterior es gracias a la capacidad que puede desarrollar cada persona de acceder a su propia vida emocional; siendo esto, a su vez, el éxodo de las capacidades intrapersonales del facilitador de mediación.

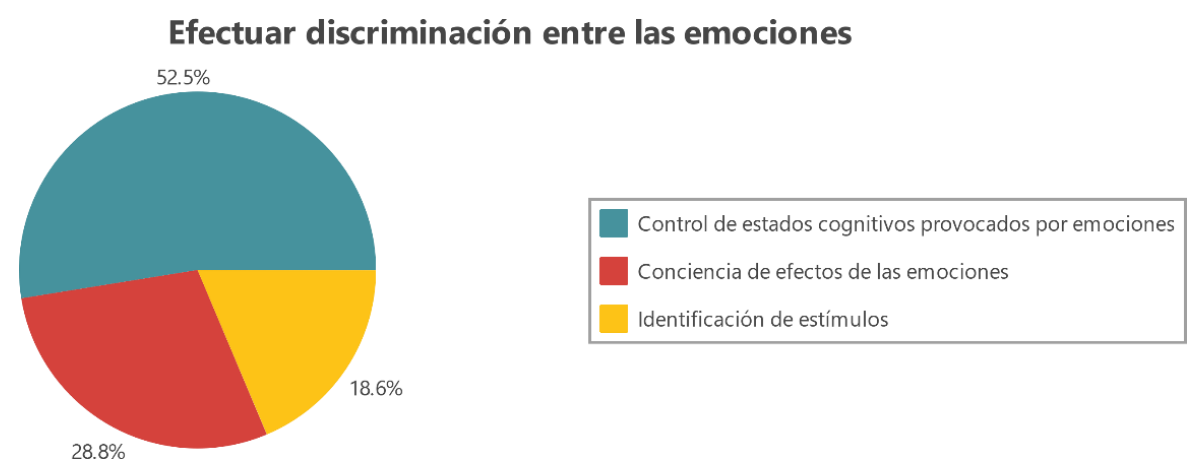

De acuerdo a las respuestas de los entrevistados, el tener acceso a la propia vida emocional es un aspecto altamente relevante para el ejercicio de la mediación. El poder darle una denominación a las emociones que se viven de manera personal a través del conocimiento de uno mismo, es un punto de partida importante para el establecimiento de la empatía al tener, al menos, una idea de la situación por la que están pasando los mediados, incluso por encontrar similitudes entre la experiencia que llevan los mediados en el conflicto y una experiencia previa; que a través de la regulación de la conducta se comprende que no se deben mezclar las emociones del mediador con la gestión del conflicto para mantener la imparcialidad, neutralidad 
y objetividad; y todo esto tiene como punto de partida el identificar los propios estados de ánimo que experimenta la persona que ejerce la profesión de mediación.

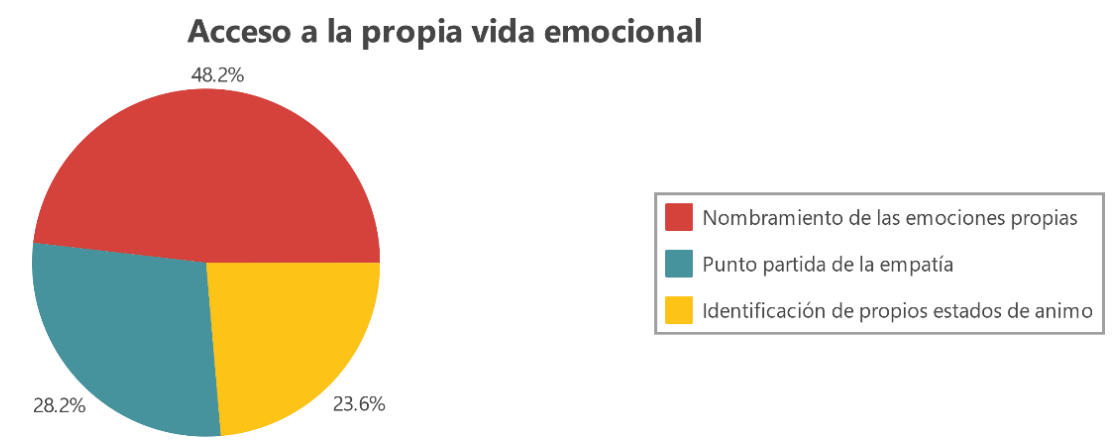

\section{CONCLUSIONES}

De acuerdo a los resultados obtenidos del análisis de las entrevistas a través del software MAXQDA para Windows, la teoría de las Inteligencias Múltiples, los elementos legales y académicos referentes a la mediación y el perfil del mediador en el ámbito familiar; consideramos viable la posibilidad de utilizar el marco de las inteligencias múltiples para estructurar un perfil de mediador familiar, complementando las competencias intrapersonales para el ejercicio profesional de la mediación considerando principalmente la propuesta de Gorjón y Sánchez (2015), quedando de la siguiente manera: 


\begin{tabular}{|c|c|c|c|c|}
\hline Fuente & Lógico-matemática & Lingüística & Intrapersonal & Interpersonal \\
\hline 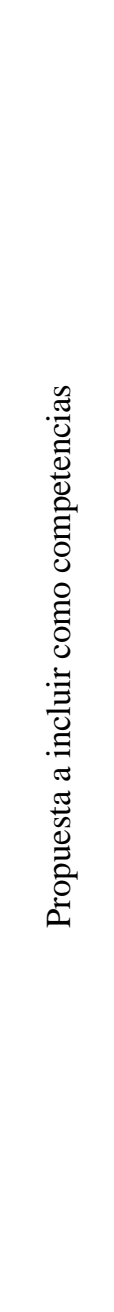 & $\begin{array}{c}\text { II. Conoce los diferentes } \\
\text { modelos de mediación y sus } \\
\text { técnicas. } \\
\text { III. Distingue entre los } \\
\text { diferentes modelos de } \\
\text { mediación y/o conciliación. } \\
\text { IV.Conoce las técnicas de } \\
\text { la mediación y de la } \\
\text { conciliación. } \\
\text { XI. Estructura el plan de } \\
\text { mediación y conciliación. } \\
\text { XII. Conoce las distintas } \\
\text { etapas procedimentales } \\
\text { desde el plan-tratamiento } \\
\text { del conflicto hasta el } \\
\text { acuerdo. } \\
\text { XVI. Definen y distinguen } \\
\text { las técnicas, teorías y } \\
\text { conceptos asociados de } \\
\text { control de crisis en un } \\
\text { conflicto. } \\
\text { XVIII. Concretan las } \\
\text { opciones que favorezcan a } \\
\text { las partes, que sean } \\
\text { susceptibles de convenir y } \\
\text { que no alteren el orden } \\
\text { público. }\end{array}$ & $\begin{array}{l}\text { V. Son capaces } \\
\text { de reestructurar } \\
\text { la comunicación } \\
\text { de las partes a } \\
\text { través de } \\
\text { técnicas de } \\
\text { comunicación. } \\
\text { VI. Desarrolla el } \\
\text { sentido de la } \\
\text { escucha activa. } \\
\text { VII. Desarrolla } \\
\text { la técnica del } \\
\text { parafraseo. } \\
\text { XIII. Son } \\
\text { capaces de } \\
\text { comprender la } \\
\text { historia del } \\
\text { conflicto para } \\
\text { detectar si es } \\
\text { susceptible de } \\
\text { iniciar la } \\
\text { mediación o la } \\
\text { conciliación. } \\
\text { XX. Elaboran el } \\
\text { acuerdo o } \\
\text { convenio en el } \\
\text { que las partes se } \\
\text { cercioran de que } \\
\text { sus pretensiones } \\
\text { han sido } \\
\text { satisfechas. }\end{array}$ & $\begin{array}{l}\text { * Conoce las } \\
\text { emociones básicas } \\
\text { del ser humano y } \\
\text { los aspectos } \\
\text { fisiológicos y } \\
\text { cognitivos que las } \\
\text { caracterizan } \\
\text { * Identifica y } \\
\text { reconoce los } \\
\text { estados } \\
\text { emocionales que se } \\
\text { le presentan antes y } \\
\text { durante la } \\
\text { mediación } \\
\text { * Son capaces de } \\
\text { regular y canalizar } \\
\text { sus emociones para } \\
\text { separarse del } \\
\text { conflicto } \\
\text { gestionado } \\
\text { * Son capaces de } \\
\text { comunicarse } \\
\text { asertivamente a } \\
\text { través del lenguaje } \\
\text { verbal y no verbal } \\
\text { de manera } \\
\text { imparcial, neutral } \\
\text { y objetiva }\end{array}$ & $\begin{array}{l}\text { I. Conoce e identifica } \\
\text { las materias que son } \\
\text { susceptibles de mediar } \\
\text { o conciliar. } \\
\text { VIII. Conoce y aplica } \\
\text { la técnica del Caucus } \\
\text { como elemento sine } \\
\text { qua non de la } \\
\text { mediación } \\
\text { conciliación. } \\
\text { IX. Conoce y aplica la } \\
\text { técnica de control de } \\
\text { la ira durante el } \\
\text { procedimiento de } \\
\text { mediación- } \\
\text { conciliación. } \\
\text { X. Conoce y aplica la } \\
\text { técnica del rapport. } \\
\text { XIV. Detecta las } \\
\text { posiciones de las } \\
\text { partes en el conflicto. } \\
\text { XV. Detecta los } \\
\text { intereses de las partes. } \\
\text { XVII. Son capaces de } \\
\text { motivar opciones para } \\
\text { la solución del } \\
\text { conflicto por parte de } \\
\text { los involucrados. } \\
\text { XIX. Son capaces de } \\
\text { sustraer los intereses y } \\
\text { necesidades mutuas de } \\
\text { los protagonistas del } \\
\text { conflicto. }\end{array}$ \\
\hline
\end{tabular}

Elaboración propia en base a (Gardner, Estructuras de la mente, 2001) y (Gorjón Gómez \& Sánchez García, 2015)

\section{REFERÊNCIAS}

De Villa Cortés, J. C. (2012). La mediación en Guanajuato. Acta Universitaria. Universidad de Guanajuato, 19-22.

García Villanueva, L., \& Vázquez de Castro, E. (2013). La mediación civil en España: luces y sombras de un marco normativo. Política y Sociedad, 71-98.

Gardner, H. (2001). Estructuras de la mente. Bogotá: Fondo de Cultura Económica Ltda. 
Gardner, H. (2001). Estructuras de la mente. Bogotá: Fondo de la Cultura Económica Ltda. Gorjón Gómez, F. J., \& Sánchez García, A. (2015). Las 101 preguntas del mediador. México:

Tirant lo Blanch.

Gorjón, F. (2015). Teoría de la impetración de la justicia. por la necesaria ciudadanización de la justicia y la paz. Comunitaria, 113-131.

Gorjón, F., \& Steele, J. (2012). Métodos alternos de solución de conflictos. México: Oxford University Press México S.A. de C.V.

Gorjón, F., Pesqueira, J., Sáenz, K., Vázquez, R., Quiroz, L., Cabello, P., . . . Steele, G. (2015).

La ciencia de la mediación. México: Tirant lo blanch México.

Igleias-Ortuño, E. (2016). Construcción jurídica del perfil profesional de la mediación en España. La razón histórica. Revista hispanoamericana de historia de las ideas, 130- 153.

Iglesias-Ortuño, E., \& Pastor-Seller, E. (2015). Las competencias para el ejercicio profesional mediador. Estudio de la actualidad formativa Catalana en materia de Mediación. Murcia, Murcia, España: Universidad de Murcia.

Instituto Interamericano para el Desarrollo Social. (2000). Diseño y gerencia de políticas y programas sociales. Eficacia, Eficiencia, Equidad y Sostenibilidad: ¿Qué queremos decir? Banco Interamericano de Desarrollo.

Jefatura del Estado (España). (2012). Ley 5/2012, de 6 de julio, de mediación en asuntos civiles y mercantiles. España. Obtenido de https://www.boe.es/buscar/pdf/2012/BOE-A-2012-9112consolidado.pdf

Macías, M. A. (2002). Las múltiples inteligencias. Psicología desde el Caribe, 27-38.

Miranzo de Mateo, S. (2010). Quiénes somos, a dónde vamos... origen y evolución del concepto de mediación. Revista de Mediación, 8-15.

Naciones Unidas. (2012). Directrices de las Naciones Unidas para una mediación eficaz.

Directrices de las Naciones Unidas para una mediación eficaz. Nueva York, Estados Unidos de América. Obtenido de https://peacemaker.un.org/sites/peacemaker.un.org/files/GuidanceEffectiveMediation_UNDP A2012\%28spanish\%29_1.pdf

Sáenz López, K. A., \& Rivera Hernandez, P. P. (2012). La mediación, concepto y ámbitos de aplicación. En D. L. Batista, H. A., R. Soler Mendizábal, J. L. Prado Maillard, \& F. J. Gorjón Gómez, Modernización de la justicia desde la perspectiva Panameña y Mexicana. Panamá: Publidisa. 
Sáenz, K., \& Téllez-Castilla, M. (2014). La entrevista en profundidad. En K. Sáenz, \& G. Tamez, Métodos y técnicas cualitativas y cuantitativas aplicables a la investigación en ciencias sociales (págs. 171-182). Ciudad de México: Tirant Humanidades.

Serrano, G. (2008). Eficacia y Mediación Familiar. Boletín de Psicología, 51-63. Serrano, G. (2008). Eficacia y Mediación Familiar. Boletín de Psicología, 51-63.

Data de Submissão: 13/03/2020

Data de Aceite: 16/04/2020 